\title{
Heating the solar corona by plasma loops
}

\author{
J. M. A. Ashbourn and L. C. Woods
}

Mathematical Institute, University of Oxford, 24-29 St Giles', Oxford OX1 3LB, UK

e-mail: ashbourn@maths.ox.ac.uk;leslie.woods@balliol.oxford.ac.uk

Received 23 October 2005 / Accepted 23 May 2006

\begin{abstract}
We investigate the heating of the corona via plasma loops. It is shown that it may be possible to maintain the high corona temperatures using plasma loops as conduits. Under certain conditions heat can flow across magnetic fields up temperature gradients, a mechanism that has been previously applied to the heating of plasma loops. A typical conduit loop is hotter than the ambient plasma in the upper chromosphere and transition layer, and is cooler than the ambient plasma in the background corona. Hence, heat enters the loop at the bottom, is transported by a combination of conduction (if there is a negative temperature gradient), convection and shock waves up the loop into the corona. Typical values show that this type of heating is sufficient to maintain both the quiet and active corona and that it also has the non-homogeneous temperature distribution observed in the lower corona. The behaviour of some brightening events seen in TRACE data support the proposed convective and shock wave mechanisms. The model offers a possible explanation of a long-standing problem, namely why the corona is so hot.
\end{abstract}

Key words. plasmas - Sun: corona - Sun: magnetic fields

\section{Introduction}

Although various theories have been advanced in an effort to explain why the corona is so much hotter than the photosphere, this problem remains unsolved (e.g. see Zirker 1993a; Aschwanden 2001, 2004); however, Schrijver \& Zwann (2000) believe that the real question is which mechanism dominates. Observations show that the heating takes place over magnetically enhanced regions and it is generally agreed that the conversion of magnetic energy into thermal energy is the most likely source of coronal energy. The mechanism for this conversion which is most often advanced is termed "reconnection". It is observed that solar magnetic fields sometimes rearrange into configurations of lower energy and during this process magnetic elements connected to one pole then reconnect to another. For this to happen, the fields lines must move across one another; however, this is only possible in the presence of either very steep gradients or the considerably enhanced resistivity generated by an instability.

In their account of coronal activity, Koutchmy et al. (1991) report that the observed temperatures of the inner corona fall into four regimes:

1. the most probable temperature, $\sim 2 \times 10^{6} \mathrm{~K}$, which matches density structures quite well;

2. temperatures of $\sim 1 \times 10^{6} \mathrm{~K}$ that probably indicate plasma in a transitory state;

3. extreme temperatures higher than $3 \times 10^{6} \mathrm{~K}$, for example above flaring regions;

4. much lower temperatures, as observed in slowly erupting prominences.

Thus the corona is largely an inhomogeneous mixture of hot and cool loops, often close together, if not occupying the same space.
Zirker (1993a) lists several observational constraints that an acceptable model of coronal heating should satisfy:

1. the corona contains a variety of large-scale structures such as plasma loops and these in turn contain considerable fine structure;

2. the coronal temperature and density distributions are highly inhomogeneous. In the quiet corona much of the plasma has a temperature near $2 \times 10^{6} \mathrm{~K}$, while in active regions some of it reaches between $8-20 \times 10^{6} \mathrm{~K}$;

3. the corona is quasi-static at large scales $\left(\sim 10^{4} \mathrm{~km}\right.$ and $30 \mathrm{~min}$ ), but exhibits fluctuations in brightness and velocity fields at smaller scales;

4. the average power requirements for the active and quiet corona are $10^{4}$ and $3 \times 10^{2} \mathrm{Wm}^{-2}$ respectively;

5. coronal magnetic field strengths are estimated at between $1-100 \mathrm{G}$.

Zirker then describes in some detail the three principal mechanisms for coronal heating, namely (i) wave heating, (ii) electric currents (mainly reconnection) and (iii) MHD turbulence heating. Each mechanism presents some difficulties in its underlying theory and also in the supporting observations, although Zirker concludes that these remain viable candidates for coronal heating. They all depend on photospheric motions at the very edge of possible resolution, for example they may result from the twisting of flux tubes in intergranular vortices, which are below the resolution of present observational techniques. In another paper, Zirker (1993b) shows that field line twisting in these vortices could supply a substantial fraction of the required power to the quiet corona. However, for the active corona it would appear to fall well short of what is required and a different mechanism would be necessary.

Another mechanism for heating the corona is the dispersal of flare energy, especially that of very small and presently unresolvable invisible flares called "nanoflares", since observable 
flares are too rare to supply the required energy. If it is believed that reconnection is the source of flare energy, then this form of heating would fall under mechanism (ii) above. However, as Zirker (1993a) observes, if nanoflares are responsible for heating the active corona, then at energies below $10^{26} \mathrm{erg}$ they must be more frequent than an extrapolation of the observed power law implies. Aschwanden (1999) has reached the same conclusion by attempting to extrapolate empirical scaling laws for the length of flare loops below the nanoflare regime and finding a lower limit in flare energy. He concludes that the energy content of the heated plasma observed in EUV, SXR and HXR flares is two to three orders of magnitude too small to account for coronal heating. On the other hand, Aschwanden (2004) concludes that the radiation losses from the quiet Sun corona - about one third of the total losses - can be accounted for by the observed nanoflare distribution.

\section{Plasma loops as conduits}

The possible role of plasma loops in directly heating the corona is worth considering. For example, if we could understand how the very hot loops with temperatures exceeding $2 \times 10^{6} \mathrm{~K}$ acquire their thermal energy, this might provide a clue to the origin of coronal heating. The location of the heating source is a first step towards understanding this. Studies of active region loops observed with TRACE (Aschwanden et al. 2001) appear to give strong support for a heat source concentrated near the bottom of the loop. Aschwanden (2001) analyzed plasma loops observed by Yohkoh in an arcade lying in the Sun's northern hemisphere in order to determine the heating scale height $\left(s_{\mathrm{H}}\right)$. He found a value of $s_{\mathrm{H}} \approx 8.4 \mathrm{Mm}$ and concluded that coronal loops are generally heated near their footpoints. This contrasts with earlier work by Priest et al. (1998), which gave a much larger value for $s_{\mathrm{H}}$ with the conclusion that the loops were more likely to be heated uniformly.

In Ashbourn et al. (2001) a new method of heating these loops was described, which depended on terms in the thermal conductivity that are second-order in the Knudsen number expansion, i.e. $\boldsymbol{q}$ is written as $\boldsymbol{q}=\boldsymbol{q}_{1}+\boldsymbol{q}_{2}+\ldots$, where $\boldsymbol{q}_{1}$ is the usual first-order heat flux known as Fourier's law and $\boldsymbol{q}_{2}$ is the second-order term, which is proportional to the scalar product of the temperature gradient, $\nabla T$ and the velocity gradient tensor, $\boldsymbol{\nabla} \boldsymbol{v}$. Normally of course we expect $\left|\boldsymbol{q}_{2}\right| \ll\left|\boldsymbol{q}_{1}\right|$, but this is not necessarily true in the presence of relatively strong magnetic fields.

The aim of this paper is to consider plasma loops with intermediate temperatures and to show that second-order thermal transport can provide a mechanism for heating parts of the corona via these loops. In our model heat actually flows upwards from the upper chromosphere and transition regions using cool flux tubes as conduits. The mechanism is illustrated in Fig. 1. In the domain below the transition region, the loop temperature is greater than that in the ambient plasma and by the secondorder mechanism (described below), heat flows up the temperature gradient and across the magnetic field into the loop. This causes a local increase in temperature, and both temperature and pressure gradients are established which transport the thermal energy by conduction, convection, and possibly shock waves up to that part of the loop high in the corona. Shock waves are likely if the loop temperature gradient is too small to remove rapidly supplied energy at the lower end of the loop.

There are two views on the nature of the corona; either the flux tubes spread with increasing height so as to fill the

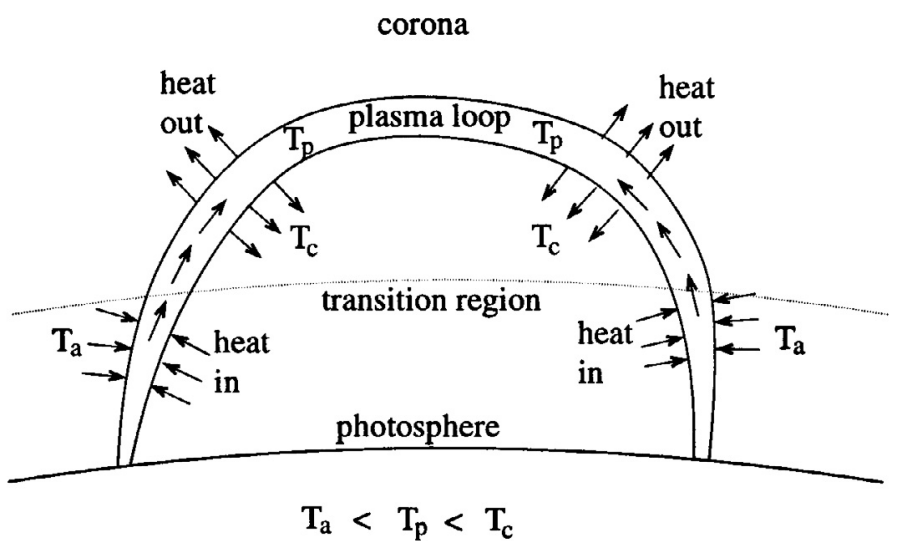

Fig. 1. Transporting heat to the corona via plasma loops.

whole of the upper corona, in which case "heating the corona" would mean merely heating the flux tubes, or else there is an background plasma lying outside the tubes which we call the "corona". With (closed) plasma loops the latter model seems to be the most appropriate and we shall adopt this geometry, but our theory applies equally to either model. Loop heating without reference to the background corona was treated in an earlier paper (Ashbourn et al. 2001).

High up in the loop the situation is the reverse of that at the bottom, that is, the temperature in the loop is less than that in the ambient corona and therefore heat now flows out of the loop into the hotter corona. We require loops hotter than the ambient temperature in the upper chromosphere, e.g. hotter than about $5 \times$ $10^{4} \mathrm{~K}$ and assuming that the corona is outside the loops, cooler than the typical coronal temperature of $\sim 2 \times 10^{6} \mathrm{~K}$. It is clear that there are such loops from the figures given in Table 2.9 of Bray et al. (1991); these fall into the category of cool loops, but near the hotter end of this type.

We would expect conduit loops to be approximately isothermal as heat supplied near the footpoints should flatten the temperature profiles within minutes (Ashbourn et al. 2001). Lenz et al. (1999) have studied temperature and density profiles in four isolated loops observed by TRACE and found constant temperatures of $\sim 1.3 \times 10^{6} \mathrm{~K}$; they conjectured that there is a class of isothermal loops distinct from those loops with temperature maxima at their apexes. Because the thermal conductivity in the electron gas parallel to the magnetic field lines is relatively large, the required fall in temperature to conduct energy up into the corona does not need to be large. Alternatively, as will be described in Sect. 5, there are TRACE observations that can be interpreted as being upward pulses of hot material for which a temperature gradient is not required.

The problem which we are concerned with here is the transference of heat upwards from the chromosphere to the corona, which leaves open the question of how the chromosphere itself acquires its energy. Wave heating of the chromosphere is rather easier to justify than wave heating of the corona (e.g. see Schrijver \& Zwann 2000) and there is also the possibility that spicules, which have properties similar to transient plasma loops, can transport heat from below the photosphere across the background temperature minimum to the chromosphere by the same mechanism as that proposed here for the chromosphere/corona stage. In addition the chromosphere is partially heated by the thermal energy returning down the steep transition layer gradient. Thus we have a heating 
circuit: chromosphere $\longrightarrow$ corona $\longrightarrow$ chromospheric heating plus radiation losses, which we plan to investigate in a later paper.

In Chapter 9 of his comprehensive text, Aschwanden (2004) arrives at heating energy requirements for replacing the energy losses from the chromosphere and corona, which are similar to those given above by Zirker. He also breaks down the process of coronal heating into 8 subprocesses which would require attention for a complete account of coronal heating; these are:

1. mechanical driver - the basic energy source in the photosphere and chromosphere consisting of fluid motions and waves;

2. electromagnetic coupling of the fluid motions into magnetic field line structures and electric currents;

3. magnetic energy storage into non-potential magnetic filaments;

4. instability - filament eruption and reconnection;

5. energy transport in several forms - thermal and non-thermal particle motions and various kinds of waves;

6. plasma heating, thermal conduction and energetic particles;

7. plasma flows - chromospheric evaporation and upflows of heated plasma;

8. plasma trap - the filling of overdense coronal loops.

However, few treatments of the coronal heating problem deal with all of these stages; the method that we are advancing in this paper does not require any of stages 1 to 4 . The creation of the magnetic flux tubes or fibres comprising a plasma loop is taken to be a phenomenon separable from the heating of the corona. Once a flux tube makes its appearance, provided it is anywhere hotter than the background photospheric or chromospheric plasma, the second-order heating mechanism takes over, i.e. we are assuming that stages 1 to 3 have occurred and we are proposing a thermal instability to replace stages 4, 5, 6 and 7. As for the initial heating required, ohmic heating on the boundaries of the fibres comprising the plasma loops would be sufficient to raise their temperature above ambient and hence trigger the thermal instability. Alternatively, as will be shown in Sect. 3, if a new plasma loop emerging from beneath the photosphere is hotter than $T_{\mathrm{cr}}=5.44 n_{\mathrm{e}}^{\frac{1}{4}}$, the thermal instability will heat this until it attains the properties of a conduit loop. If the ambient corona is cooler than such a loop and the conduit process does not occur, the loop will retain its thermal energy; if it continues to be heated near its base, a thermal instability will result and the corona will be heated directly perhaps via a flare, a possibility which we will consider in another paper. Presently, it is the maintenance of coronal temperatures which is of concern to us here.

\section{Cross-field energy transport}

An account of second-order heat flux in a magnetoplasma is given in Woods (2004), where it is shown that at large values of $\varpi=\omega_{\mathrm{c}} \tau$, where $\omega_{\mathrm{c}}$ is the cyclotron frequency and $\tau$ is the collision interval, the heat flux vector $\boldsymbol{q}_{2}$ is related to the deviator of the fluid velocity gradient and the magnetic induction $\boldsymbol{B}$ by

$\boldsymbol{q}_{2}=-\frac{5 k_{\mathrm{B}} p}{2 Q B^{2}} \tau \boldsymbol{B} \times \stackrel{\circ}{\nabla} \boldsymbol{v} \cdot \boldsymbol{\nabla} T$

where $k_{\mathrm{B}}$ is Boltzmann's constant, $p$ is the pressure, and $Q$ is the particle electric charge. This is the basic equation for secondorder thermal transport across strong magnetic fields and it applies to both the ion and electron gases, although its value is largest for the electron gas. It was first developed to explain the large energy losses from tokamaks (Woods 2006).

By evaluating $\boldsymbol{\nabla} \boldsymbol{v}$ in a flux tube with axial symmetry we find that in the electron gas the second-order radial heat flux is (Woods 2004)

$Q_{\mathrm{er}}^{(2)}=-\frac{5 k_{\mathrm{B}} p_{\mathrm{e}}}{2 e B} \tau_{\mathrm{e}} H_{\mathrm{e}} T_{\mathrm{e}}^{\prime} \quad\left(H_{\mathrm{e}} \equiv \frac{1}{2} r\left(v_{\mathrm{e} \theta} / r\right)^{\prime} b_{z}-\frac{1}{2} v_{\mathrm{ez}}^{\prime} b_{\theta}\right)$,

where $v_{\mathrm{e} \theta}$ and $v_{\mathrm{ez}}$ are the azimuthal and axial components of the electron fluid velocity respectively, $p_{\mathrm{e}}$ is the electron fluid pressure, $b_{z}=B_{z} / B, b_{\theta}=B_{\theta} / B$, and the prime (') denotes the radial gradient. Adopting the approximation $\boldsymbol{v}_{\mathrm{e}}=-\boldsymbol{j} / e n_{\mathrm{e}}$, where $\boldsymbol{j}$ is the electric current density, and taking the limit $r \rightarrow \infty$, we obtain the value $H_{\mathrm{e}}=-j^{2} /\left(2 e n_{\mathrm{e}} B\right)$ for planar geometry.

With first-order thermal transport at large values of $\varpi=$ $\tau e B / m$, the ion thermal conductivity is dominant in radial heat flux $\left(q_{\text {ir }} \approx-\kappa T_{\mathrm{i}}^{\prime} / \varpi_{\mathrm{i}}^{2}\right)$, whereas with the second-order terms it follows from Eq. (1) that the electron thermal conductivity dominates. Hence

$q_{r}=\left\{\frac{5 k_{\mathrm{B}} p_{\mathrm{e}} n_{\mathrm{e}} \tau_{\mathrm{e}}}{\left(2 e n_{\mathrm{e}} B\right)^{2}} \mu_{0} j^{2}\right\} \frac{\partial T_{\mathrm{e}}}{\partial r}-\left\{\frac{2 k_{\mathrm{B}} p_{\mathrm{i}} m_{\mathrm{i}}}{e^{2} B^{2} \tau_{\mathrm{i}}}\right\} \frac{\partial T_{\mathrm{i}}}{\partial r}$.

Equation (3) is a good approximation for flux tubes that carry electric currents in narrow annular regions on their surfaces, which is a likely occurrence since the reduction in resistivity due to ohmic heating tends to channel electric currents into streamers.

From Eq. (3) we find that the heat will flow inwards, i.e. up the temperature gradient, provided that

$\frac{T_{\mathrm{ep}}}{T_{\mathrm{ip}}} \frac{5 n_{\mathrm{e}}^{2} \tau_{\mathrm{e}} \tau_{\mathrm{i}}}{8 n_{\mathrm{e}}^{3} m_{\mathrm{i}}} \mu_{0} j^{2}>1$,

where the subscript $\mathrm{p}$ denotes conditions in the plasma loop and we have assumed that the temperature gradients are proportional to the temperatures. Under typical plasma loop conditions (described below) we find that $\tau_{\mathrm{e}} \sim 10^{-5} \mathrm{~s}$, whereas the timescale for the transfer of electron energy to the ions is $\sim 0.02 \mathrm{~s}$. However, for phenomena on the time-scale of interest to us, we may assume that $T_{\mathrm{ip}} \approx T_{\mathrm{ep}}$ and write the condition (in MKS units) as

$j>j_{\text {cr }} \equiv 2.16 \times 10^{-17} \ln \Lambda\left(n_{\mathrm{e}} / T_{\mathrm{e}}\right)^{3 / 2}$,

where $\ln \Lambda \quad\left(=22.81+\ln T_{\mathrm{e}}-0.5 \ln n_{\mathrm{e}}\right.$ provided $T_{\mathrm{e}}>1.16 \times$ $\left.10^{5} \mathrm{~K}\right)$, is the Coulomb logarithm. In the following we shall set $\ln \Lambda=20$, which is a close enough approximation for the thermodynamic conditions in the plasma loops of interest here.

To satisfy Eq. (4) strong currents are required. Under coronal conditions the magnetic diffusivity is several orders of magnitude larger than the thermal diffusivity, which means that the heat from ohmic dissipation remains relatively local and raises the temperature of the electrons along the path of the current. Hence the resistivity is reduced, which allows the current density $j$ to increase up to its maximum value, say $j_{\max }$. This value is set by the two-stream instability (e.g. see Krall \& Trivelpiece 1973, p. 477), which sets a limit $C$ to the relative speed $\left|\boldsymbol{v}_{\mathrm{e}}-\boldsymbol{v}_{\mathrm{i}}\right|$, where $\left(k_{\mathrm{B}} T_{\mathrm{e}} / m_{\mathrm{i}}\right)^{\frac{1}{2}}<C<\left(k_{\mathrm{B}} T_{\mathrm{e}} / m_{\mathrm{e}}\right)^{\frac{1}{2}}$. At $T_{\mathrm{e}} \approx T_{\mathrm{i}}, C$ is near the upper limit, while if $T_{\mathrm{e}} \gg T_{\mathrm{i}}, C$ is just above the lower limit.

When the instability is first switched on, turbulence is quickly generated, which in turn greatly increases the resistivity and reduces the current until it falls below $j_{\max }$ and the instability is switched off. The sharp fall in resistivity allows $j$ to 
increase beyond $j_{\max }$ again, and so on; the marginal stability condition is thus maintained in this manner, and since the transport is in the electron gas and the electrons are very quickly heated compared to the ions, on the time-scale of the actual transport process, $T_{\text {ip }} \ll T_{\text {ep }}$, which then allows us to adopt the relation

$j_{\max }=e n_{\mathrm{e}}\left(k_{\mathrm{B}} T_{\mathrm{e}} / m_{\mathrm{i}}\right)^{\frac{1}{2}} \approx 1.46 \times 10^{-17} n_{\mathrm{e}} T_{\mathrm{e}}^{\frac{1}{2}}$.

It follows that heat will flow up the temperature gradient if $j_{\max }>j_{\text {cr }}$ or from Eq. (4), $T_{\mathrm{e}}>T_{\text {cr }}=5.44 n_{\mathrm{e}}^{\frac{1}{4}}$, and Eq. (3) may be expressed

$q_{r}=\frac{5 k_{\mathrm{B}} p_{\mathrm{e}} n_{\mathrm{e}} \tau_{\mathrm{e}}}{\left(2 e n_{\mathrm{e}} B\right)^{2}} \mu_{0} j_{\max }^{2} \frac{\partial T_{\mathrm{e}}}{\partial r}\left(1-\frac{T_{\mathrm{cr}}^{4}}{T_{\mathrm{e}}^{4}}\right) \quad\left(T_{\mathrm{cr}}=5.44 n_{\mathrm{e}}^{\frac{1}{4}}\right)$.

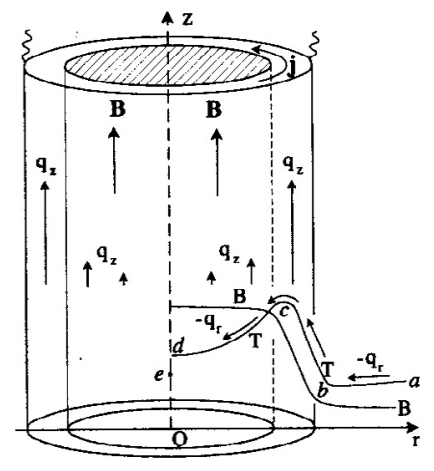

(a) Lower atmosphere

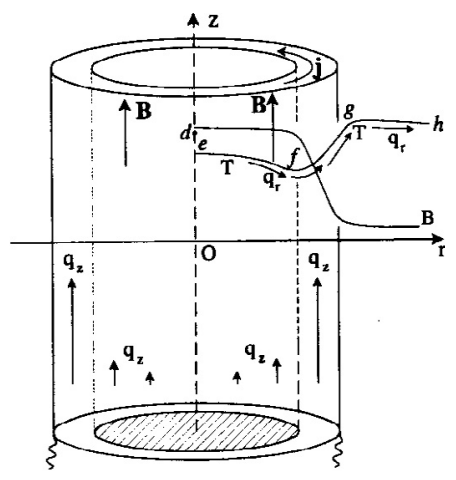

(b) Corona
Fig. 2. Temperature and magnetic field profiles for a) the lower atmosphere and b) the corona.

\section{Application to coronal heating}

At the end of Sect. 1 we identified the type of plasma loop that is required for the mechanism to remove heat from the chromosphere and most of the transition layer and then transfer it to the corona above. For example, a loop at a temperature $T_{\mathrm{p}}=0.5 \times 10^{6} \mathrm{~K}$ would do this, being hotter than ambient between the chromosphere and a point above the mid-point of the transition layer and cooler than ambient in the corona. However, there remains the question of exactly which temperature to adopt in Eq. (6) - whether the slowly varying value in the loop, $\left(T_{\mathrm{p}}\right)$, or some average between the loop temperature and the ambient plasma temperature, say $T_{\mathrm{a}}$. At the lower end of the loop the path of the electric current which drives the inward heat flux is likely to be much closer to the higher temperature $T_{\mathrm{p}}$, where the resistivity is smaller, than it is to lie in the ambient plasma. Since our objective is simply to show that the corona could be heated by the mechanism shown in Fig. 1, we shall adopt the value $T_{\mathrm{p}}=10^{6} \mathrm{~K}$. This temperature and $n_{\mathrm{e}}=2 \times 10^{15} \mathrm{~m}^{-3}$ (see Aschwanden 2004, p. 204) gives $T_{\mathrm{cr}} \approx 3.6 \times 10^{4} \mathrm{~K}$, so that the inequality for a thermal instability is satisfied, allowing us to use Eq. (6) to determine the flux of thermal energy into the loop. At the higher end of the plasma loop, both the loop temperature and the electron number density will have fallen a little, so we expect no change to the situation described above, except that the heat will now flow out from the loop into the hotter corona.

Since it is reasonable to assume that in general the length of the loop in the corona will somewhat exceed the length of the loop in the lower atmosphere, the rate of heat flow through the system will not be constrained in the corona. Also there is a change that ensures that this is certainly the case. A dipole model of the magnetic field which fits the observed loop structures well gives a variation with height $h$ according to the empirical law (see Aschwanden 2004, p. 202)

$$
B(h)=B_{0}\left(1+\frac{h}{h_{\mathrm{D}}}\right)^{-3} \quad\left(h_{\mathrm{D}}=74 \times 10^{6} \mathrm{~m}\right),
$$

where typical cool loops have $h_{\max } \approx 46 \mathrm{Mm}$ and typical hot loops have $h_{\max } \approx 120 \mathrm{Mm}$ (Bray et al. 1991). Thus up to the maximum height, due to its $B_{\mathrm{g}}^{-3}$ dependence, $Q_{r}$ will be increased by orders of magnitude. It follows that in order to determine the rate at which the corona is being heated, it is sufficient to just consider the rate at which the heat enters the loop in the lower atmosphere.

Figure 2 illustrates typical $(T, r)$ paths, which are labeled $a b c d$ in the lower atmosphere, de in the plasma loop, and $e f g h$ in the corona. Energy is transferred by conduction from point $a$ up to point $h$ with the plasma loop acting as the conduit. We have illustrated the conduit by the path $d e$, but of course any path within the loop parallel to the magnetic field would serve the same purpose. Second-order transport in the electron fluid occurs along the paths $\boldsymbol{b} \boldsymbol{c}$ and $\boldsymbol{f g}$, which cross the narrow region through which the electric current passes. The cross-field energy transport along $\boldsymbol{c d}$ and $\boldsymbol{e} \boldsymbol{f}$ occurs mainly in the ion gas - the second term on the right-hand side of Eq. (3) - while any axial conduction along $\boldsymbol{d e}$ is mostly in the electron gas. Convective and shock wave transport along the axis will be mainly in the ion component of the plasma. However, these distinctions are not important since at this stage the macroscopic time-scales are long enough for thermal equilibrium between the two gases to be established. We start by determining the rate at which heat flows inwards across the unit area of a typical loop. We divide the formula for $q_{r}$ in Eq. (6) into three factors, the first of which is

$F_{1}=\frac{5 k_{\mathrm{B}} p_{\mathrm{e}} n_{\mathrm{e}} \tau_{\mathrm{e}}}{4 e^{2} n_{\mathrm{e}}^{2} B^{2}}=\frac{5 k_{\mathrm{B}}^{2}}{4 e^{2}} \frac{2.75 \times 10^{5}}{\ln \Lambda} \frac{T_{\mathrm{e}}^{\frac{5}{2}}}{n_{\mathrm{e}} B^{2}} \approx 1.28 \times 10^{-4} \frac{T_{\mathrm{e}}^{\frac{5}{2}}}{n_{\mathrm{e}} B^{2}}$.

The second factor is

$F_{2}=\mu_{0} j_{\max }^{2} \frac{\partial T_{\mathrm{e}}}{\partial r}=\mu_{0} j_{\max }^{2} \frac{\partial B}{\partial r} \frac{\partial T_{\mathrm{e}}}{\partial B} \approx \mu_{0}^{2} j_{\max }^{3} \frac{\left[T_{\mathrm{e}}\right]}{B}$,

where $\left[T_{\mathrm{e}}\right]$ is the jump in $T_{\mathrm{e}}$ across the current sheet, the width $\lambda_{\mathrm{B}}$ of which is estimated from $\mu_{0} j_{\max }=\partial B / \partial r \sim B / \lambda_{\mathrm{B}}$. By Eq. (5)

$F_{2}=4.85 \times 10^{-63} n_{\mathrm{e}}^{3} T_{\mathrm{e}}^{3 / 2}\left[T_{\mathrm{e}}\right] / B$.

Thus

$q_{r}=6.19 \times 10^{-67} \frac{n_{\mathrm{e}}^{2} T_{\mathrm{e}}^{4}}{B^{3}}\left[T_{\mathrm{e}}\right]\left(1-T_{\mathrm{cr}}^{4} / T_{\mathrm{e}}^{4}\right)$.

If we let $n_{\mathrm{e}}=10^{16} n_{16}, T_{\mathrm{e}}=10^{5} T_{5}, B=10^{-4} B_{\mathrm{g}}$, then the expression for $q_{r}$ becomes

$q_{r}=6.19 \times 10^{2} n_{16}^{2} T_{5}^{4}\left[T_{5}\right] B_{\mathrm{g}}^{-3}\left(1-T_{\mathrm{cr}}^{4} / T_{\mathrm{e}}^{4}\right)$.

For the cool loops of interest the variables $n_{16}$ and $T_{5}$ are of the order of unity and the coronal value of the magnetic field $B_{\mathrm{g}}$ is between 4 and $45 \mathrm{G}$ (Bray et al. 1991). For a typical value of $B_{\mathrm{g}}$ in the chromosphere we shall take $B_{\mathrm{g}} \approx 80 \mathrm{G}$ (cf. the range of values for active regions given in Fig. 5.9 of Aschwanden 2004). The strong dependence of $q_{r}$ on $T$ and $B_{\mathrm{g}}$ means that the chance of obtaining accurate typical values for the heat flux is small, so 
the best we can expect from the theory is to establish that the proposed mechanism is easily able to supply enough heat to the corona to match the losses from it by radiation and conduction.

An even greater uncertainty is that involved in determining the total area through which heat is able to enter all of the suitable plasma loops. Zirker (1993b) had to solve a similar problem, although in his case he needed an estimate of the crosssectional area at the base of the loops, since in his vortex model the energy could only enter through the base. He chose a filling factor $f$ for the photospheric flux of $1 \%$, which includes some allowance for the lack of resolution of the footpoint diameters. We shall adopt this value here, but it should be noted that both $f$ and $B$ will vary considerably from active region loops to quiet region loops.

If we let $d$ denote the average diameter of a cool plasma loop and denote by $\mathcal{L}$ the average length of a loop below the corona which is able to absorb energy from its surroundings, then the total surface area across which this occurs is $\pi d \mathcal{L} N$, where $N$ is the total number of loops involved at a given time. With $f$ equal to the flux tube filling factor, $N=4 f A_{\odot} / \pi d^{2}$, where $A_{\odot}$ is the surface area of the Sun. The ratio of the loop area across which heat is being absorbed to that of the solar surface is therefore $4 f \mathcal{L} / d$. If we let $Q_{r}$ be the average energy flux emitted into the corona and assuming that this is balanced by the heat absorbed from the lower atmosphere, we have

$$
\begin{aligned}
Q_{r} & =-(4 f \mathcal{L} / d) q_{r} \\
& \approx 2.5 \times 10^{3}(f \mathcal{L} / d) n_{16}^{2} T_{5}^{5} B_{\mathrm{g}}^{-3}\left(1-T_{\mathrm{cr}}^{4} / T_{\mathrm{e}}^{4}\right),
\end{aligned}
$$

where we have adopted the approximation $\left[T_{\mathrm{e}}\right] \approx-T_{\mathrm{e}}$, i.e. we have ignored the relatively small temperature outside the plasma loop. Most of the variables appearing in this equation are not known observationally with any accuracy.

For $\mathcal{L}$ we shall take $5 \times 10^{6} \mathrm{~m}$, which is roughly twice the distance from the upper chromosphere to the lower corona (allowing for both sides of the loop). The diameter of the loop should be the average diameter of the magnetic threads comprising a typical plasma loop rather than the diameter of the loop itself. The greater the bifurcation of the magnetic field within the plasma loop, the more effective the absorption of energy from the ambient plasma will be. In Ashbourn et al. (2001), for hot loops with steadily increasing temperatures up to a maximum at the top of the loop, a rough estimate of the diameter was $256 \mathrm{~km}$; in this paper, following Zirker (1993b) we shall adopt the value $d=200 \mathrm{~km}$. For the loop temperature we shall take a value in the upper transition layer, say $T_{\mathrm{p}}=6 \times 10^{5} \mathrm{~K}$; for $B_{\mathrm{g}}$ we have the estimate $80 \mathrm{G}$, and for $n_{\mathrm{e}}$ the value $n_{\mathrm{e}}=5 \times 10^{16} \mathrm{~m}^{-3}$ is appropriate (see Bray et al. 1991, Table 2.9). Substituting these values into Eq. (9), we find that $T_{\mathrm{cr}}^{4} / T_{\mathrm{e}}^{4}$ is negligible and we obtain the estimate $Q_{r}=1280 \mathrm{~W} \mathrm{~m}^{-2}$, which lies in the estimated range cited in the Introduction, namely $300 \mathrm{~W} \mathrm{~m}^{-2}$ for the quiet corona and $10000 \mathrm{~W} \mathrm{~m}^{-2}$ for the active corona. Due to the sensitivity of $Q_{r}$ to temperature changes, an increase in temperature by only $51 \%$ is sufficient to increase $Q_{r}$ to the active corona value. Electron number densities are also rather larger in active regions.

For an accurate determination of $Q_{r}$, we need to start with the temperature $T_{\mathrm{a}}$ and determine the intermediate temperatures at the other points from the expressions for conductivity and the final temperature value required, $T_{\mathrm{c}}$. Also, if the temperature of the loop becomes comparable with that of the ambient corona, the energy lost to the corona from the loop will be too small to compensate for the energy input in the lower atmosphere and a thermal instability will result, which may develop into a flare.
The theory appears to be in qualitative agreement with observation, but the many uncertainties in the observed values make pursuing an "accurate" calculation of doubtful value. It follows that the heat entering the loop remains largely in surface layer from the values of the thermal diffusivities, $\left(\chi=\kappa /\left(1.5 k_{\mathrm{B}} n_{\mathrm{e}}\right)\right)$, which are as follows. If we let $\chi_{\perp 1}$ and $\chi_{\perp 2}$ denote the first and second order perpendicular diffusivities, then from Eq. (9) we find that

$$
\begin{aligned}
& \chi_{\perp 2}=\frac{5 k_{\mathrm{B}} n_{\mathrm{e}} \tau_{\mathrm{e}} T_{\mathrm{e}}}{e^{2} n_{\mathrm{e}}^{2} B^{2}} \mu_{0} j_{m}^{2}=5.19 \times 10^{4} T_{5}^{7 / 2} B_{\mathrm{g}}^{-2}, \\
& \chi_{\perp 1}=\frac{4 k_{\mathrm{B}} m_{\mathrm{i}} T_{\mathrm{i}}}{3 e^{2} B^{2} \tau_{\mathrm{i}}}=4.55 \times 10^{3} n_{16} T_{5}^{-1 / 2} B_{\mathrm{g}}^{-2}, \\
& \chi_{\|}=\frac{3.16 k_{\mathrm{B}}}{1.5 m_{\mathrm{e}}} T_{\mathrm{e}} \tau_{\mathrm{e}}=1.39 \times 10^{8} T_{5}^{5 / 2} n_{16}^{-1},
\end{aligned}
$$

of which the last, $\chi_{\|}$, follows from the standard expression for the parallel thermal conductivity, which is dominated by the electrons. With $n_{16}=5, T_{5}=6$, and $B_{\mathrm{g}}=80 \mathrm{G}$, we find from these formulae that $\chi_{\|} \gg \chi_{\perp 2} \gg \chi_{\perp 1}$. It is clear that after the heat has entered the loop through the action of the surface electric currents $\left(\chi_{\perp_{2}}\right)$, its further radial penetration is inhibited in the usual way by the magnetic field $\left(\chi_{\perp 1}\right)$ so that as indicated by $q_{z}$ in Fig. 2, it is largely either conducted away in a surface layer $\left(\chi_{\|}\right)$ or removed by convection in this layer, leaving the interior of the loop relatively cool. Observations of the EUV emission from an active region loop by Roussel-Dupré et al. (1984) and their interpretations support this configuration of a thin hot sheath on a cool loop. The authors inferred that the emission originated from very thin sheaths less than $1 \mathrm{~km}$ thick surrounding an (assumed) cool core.

\section{Unsteady conditions}

So far it has been assumed that the heat transfer in and out of the conduit loop is a steady process, but due to the strong dependence of $q_{r}$ on the temperature, this is unlikely to be the case. If we let $\mathcal{S}$ denote the surface layer of a loop, then if ohmic heating in $\mathcal{S}$ can increase the temperature of the layer above $T_{\text {cr }}$, by Eq. (9) the heat will begin to flow inwards, further heating the layer and changing the loop into a conduit loop. At a typical point $c$ shown in Fig. 2 a the first right-hand term in Eq. (3) falls to zero as the current density $j$ vanishes, and heat is transferred a little further into the loop down the temperature gradient by the ion conductivity term, which is only weakly dependent on $T$. A small increase in the temperature on the current-dominated interval $b c$ greatly increases the heat arriving at $c$, and there will be a short time delay while the ambient plasma at $c$ is heated sufficiently to increase the radial and axial temperature gradients, and from there to transfer this additional heat both further into the loop and along it up into the corona region.

The fluctuation in transporting the thermal energy will rapidly heat the loop at $c$, causing the loop to "brighten", and the sudden increase in pressure may be sufficient either to launch a shock wave traveling around the loop or to accelerate an annular slug of plasma along the field lines. A few minutes later, when this additional heat arrives at the corona via the conduit loop, a further brightening can be expected in the interval $g h$ shown in Fig. 2b. Also, during the transfer of the heat up the loop, almost all of it will be near the surface of the loop, thus we can expect the brightening to appear to extend between points $c$ and $g$, traveling at a speed in excess of the speed of sound. Of course the 
"points" $a$ through to $h$ are really representative points on surfaces, so we are describing a brightening of an annular sleeve at a lower position on the loop, which then extends upwards by a distance $\mathcal{L}_{\|}$say, to a similar sleeve higher in the corona.

The heating of the loop occurs at the expense of the energy of the plasma outside the loop, where the conductivity, being proportional to $T^{5 / 2}$, is rather less than that parallel to the magnetic field within the loop. Considering the conditions at point $b$ in Fig. 2a, we see that a drop in temperature at this point will increase the gradient along $\boldsymbol{a} \boldsymbol{b}$ and by increasing $\left[T_{\mathrm{e}}\right]$ in Eq. (7) it will also increase the flow of heat along $\boldsymbol{b c}$ and from there parallel to $\boldsymbol{d e}$. Thus additional heat will begin to flow inwards towards the loop, but at an insufficient rate to make up for the increased heat flux along the loop, this discrepancy then generating an unsteadiness in the loop heating. After some time the temperature at $b$ will be increased and by reducing $\left[T_{\mathrm{e}}\right]$ in Eq. (7) it will check the loop heating and so on.

Ireland et al. (1999) have studied 27 small brightening events found in TRACE observations, their purpose being to look for coronal heating mechanisms operating at high cadence and away from active regions. They found bright points not more than 5 arcsec in diameter which were related to other bright points typically about 10 arcsec distant, and they described what they termed "complex" events involving two bright points in which bright material appeared to move from one point to the other. The peak brightenings ranged from about three times the background to $20 \%$ of the background and the volume covered by the motion between the bright points (termed the "extension" volume) remained bright typically for about $90 \mathrm{~s}$. They observed that some extensions, whether between bright points or from a bright point to a dark region at which the extension ends, appeared to be small and discrete which they considered to be due either to the motion of material or to the motion of some kind of heating event moving along the structure which they believed to be a magnetic loop. They found no clear evidence of magnetic cancelation for any of the events. Also, the speed of transmission of the heating event was not very different from the speed of sound in the corona. This brightening event phenomenon could be explained by unsteady heating in the conduit model described in this paper. The pairing of bright points in complex events may correspond to the regions of the loop where heat is transferred into and out of the loop; more observations of the type previously made by Ireland et al. (1999) would be very helpful to resolve this issue. Schrijver et al. (1999) have studied TRACE data and drawn a number of interesting conclusions concerning the heating of loops and of the corona. In particular they conclude that:

i) the corona is comprised predominantly of thin loops that continually evolve and which are heated for minutes to tens of minutes;

ii) heating occurs predominantly within the first 10 to $20 \mathrm{Mm}$ from the loop footpoints;

iii) coronal heating can turn on and off in a time-scale of minutes or less along field line bundles;

iv) there is a relatively smooth evolution of the location of the heating over periods of at least tens of minutes;

v) upward moving pulses of hot material imply that the low altitude heating is strongly modulated on time-scales of minutes or less;

vi) in addition to the injection of heat from below, there is rapid cooling of the tops of loops;

vii) the EUV observations show that cool plasma, no hotter than about $20000 \mathrm{~K}$, also appears to be moving upwards.
There is another phenomenon which appears to have some connection with the mechanism of conduit transport, namely the peculiar EUV emission termed "moss". Mixed in with a bright, reticulated pattern of emission, there are dark "holes" of a similar size giving the whole a spongy appearance. Moss is confined to a layer about $1 \mathrm{Mm}$ thick with a base height about $1.5-2 \mathrm{Mm}$ above the photosphere. Berger et al. (1999) have described moss as being due to thermal conduction in hot, relatively high pressure coronal loops down to their footpoint regions. The holes, which interact with the EUV elements on time-scales of $10 \mathrm{~s}$, are interpreted as being cool jets or spicules.

So far we have been concerned with the transport of heat upwards. However, there will be a height $h$ in the lower atmosphere where the loop conditions satisfy $T_{\mathrm{cr}} \approx T_{\mathrm{e}}$ and below which $T_{\mathrm{cr}}>T_{\mathrm{e}}$. By Eq. (8) the absorption of heat from the loop environment will cease and the surface temperature of the loop will have a gradient that conducts the heat above $h$ down towards the footpoints. The cool jets in moss might result from the removal of energy from the loop environment above $h$. The diffusive time-scales which can be deduced from Eq. (12) are of the order of seconds or less. These descriptions of loop heating are in qualitative agreement with the conduit loop model.

\section{Conclusions}

The model proposed in this paper offers a possible explanation of a long-standing problem, namely why the corona remains so hot. The mechanism is illustrated in Fig. 1 and depends on the dominance of second-order transport in strong magnetic fields. Heat flows across the magnetic field up the temperature gradient, being absorbed by the relatively hot loops in the lower atmosphere and is then either emitted into the hotter ambient coronal plasma or simply considered as being part of the corona.

Our model requires the presence of loops in the corona with temperatures which are less than the ambient value of $\sim 2 \times 10^{6} \mathrm{~K}$ cited by Koutchmy et al. (1991), and this condition is met with both cool loops and some flare loops (Bray et al. 1991). The existence of loops with temperatures well in excess of $3 \times 10^{6} \mathrm{~K}$ could be explained by a rate of heating in the chromospheric region which is rapid enough to raise the loop temperature above the coronal value, after which our mechanism creates a serious thermal instability. Bray et al. (1991) give a temperature range for flare loops that has an upper limit above $10^{7} \mathrm{~K}$, which appears to support this process.

With regard to the five observational constraints made by Zirker (1993a) and listed in the Introduction, we note that the model described in this paper is in agreement with these. There is also the evidence of loop heating given by Aschwanden (2001), namely that the heating occurs low in the loops; our model supplies the loop with heat in the lower atmosphere and meets this observational test. It is difficult to be certain about the nature of the bright points observed by Ireland et al. (1999), but they seem to have some of the properties required for our heat transfer mechanism - the extensions are upwards from the chromosphere or lower corona, they are heating events without any evidence of magnetic cancelation that could cause localized heating, and the speed of transmission is about the same as that of a weak shock wave. The TRACE observations analyzed by Schrijver et al. (1999) also support the model, particularly (ii) and (vi), i.e. heating at the bottom and cooling at the top of the loops.

While the sensitivity of the transport process on the temperature and magnetic field strengths makes it difficult to obtain an 
accurate quantitative theory, the model is in qualitative agreement with the observations.

Acknowledgements. J.M.A.A. is supported by a Royal Commission for the Exhibition of 1851 Research Fellowship.

\section{References}

Aschwanden, M. J. 1999, Sol. Phys., 190, 233

Aschwanden, M. J. 2001, ApJ, 559, L171

Aschwanden, M. J., 2004, Physics of the Solar Corona: an Introduction (Springer)

Aschwanden, M. J., Nightingale, R. W., \& Alexander, D. 2000, ApJ, 541, 1059

Aschwanden, M. J., Poland, A. I., \& Rabin, D. M. 2001, ARA\&A, 39, 175

Ashbourn, J. M. A., Woods, L. C., \& Bingham, R. 2001, ApJ, 553, 941

Bray, R. J., Cram, L. E., Durrant, C. J., \& Loughhead, R. E. 1991, Plasma Loops in the Solar Corona (Cambridge: Cambridge University Press)
Berger T. E., De Pontieu, B., Fletcher, L., et al. 1999, Sol. Phys., 190, 400

Ireland, J., Wills-Davey, M., \& Walsh, R. W. 1999, Sol. Phys., 190, 207

Koutchmy, S., Zirker, J. B., Steinolfson, R. S., \& Zhugzda, J. D. 1991, Solar Interior and Atmosphere, ed. A. N. Cox, W. C. Livingston, \& M. S. Matthews (Arizona: Univ. of Arizona Press)

Krall, N. A., \& Trivelpiece, A. W. 1973, Principles of Plasma Physics (New York: McGraw-Hill Book Company)

Lenz, D. D., DeLuca, E. E., Golub, L., Rosner, R., \& Bookbinder, J. A. 1999, ApJ, 515, L155

Priest, E. R., Foley, C. R., Heyvaerts, J., et al. 1998, Nature, 393, 11 June

Roussel-Dupré, R., Wrathhall, J., Nicholas, K. R., et al. 1984, ApJ, 278, 428

Schrijver, C. J., \& Zwann, C. 2000, Solar and Stellar Magnetic Activity (Cambridge: Cambridge University Press)

Schrijver, C. J., Title, A. M., Berger, T. E., et al. 1999, Sol. Phys., 187, 261

Woods, L. C. 2004, Physics of Plasmas (Weinheim: WILEY-VCH)

Woods, L. C. 2006, Theory of Tokamak Transport (Weinheim: WILEY-VCH)

Zirker, J. B. 1993a, Sol. Phys., 148, 43

Zirker, J. B. 1993b, Sol. Phys., 147, 47 